\title{
Zur Frage der Energiefortleitung in Peptidketten ${ }^{1}$
}

\author{
Von W. Broser und W. Lautsch \\ Aus dem Institut für organische Chemie der Freien Universität Berlin, Berlin-Dahlem \\ (Z. Naturforschg. 11 b, 453-460 [1956]; eingegangen am 18. Februar 1956)
}

\begin{abstract}
Die photochemische Spaltung der Kohlenoxyd-Hämochromogene der Fermentmodelle [Mesohämin-IX-poly-DL-(phenylalanin-glutaminsäure) und Cytohämin-poly-L-(phenylalanin-glutaminsäure)] verläuft bei Einstrahlung in die Banden der Wirkungsgruppen mit der Quantenausbeute eins. Bei Einstrahlung in die Bande des Phenylalaninrestes wird ein Teil der von diesem Rest absorbierten Energie an der prosthetischen Gruppe wirksam. - Es werden verschiedene Mechanismen des Energietransportes in der Polypeptidkette diskutiert, wobei der Peptidwendel besondere Beachtung geschenkt wird.
\end{abstract}

$\mathrm{F}$ ür die Energiefortleitung in Eiweißstoffen sprechen eine große Zahl von Beobachtungen ${ }^{2}$, von denen hier nur die Identität von Wirkungs- und Absorptionsspektren der Kohlenoxyd-Verbindung des Warburgschen sauerstoff-übertragenden Fermentes auch im Bereich der Eiweißabsorption ${ }^{3}$ und die Leitfähigkeitsbefunde an Gelatine-Farbstoffphosphoren ${ }^{4}$ erwähnt seien.

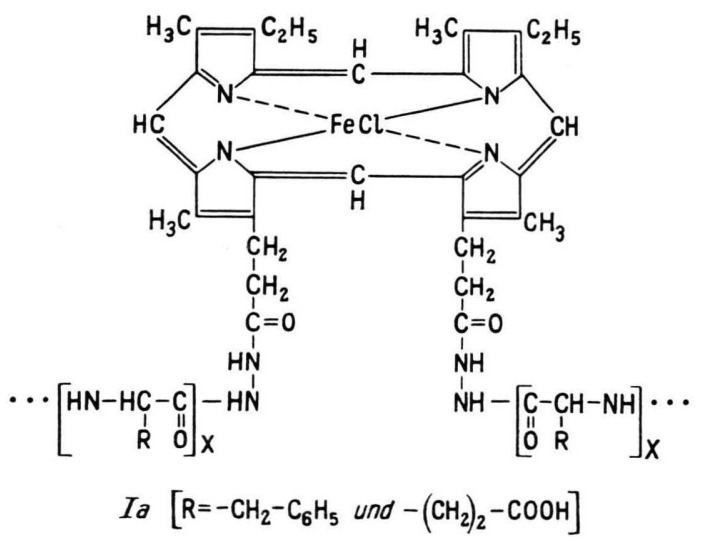

Es schien uns nun von besonderem Interesse, zu prüfen, ob derartige Energieleitungs-Effekte auch bei unseren oxydatisch wirksamen Fermentmodellen ${ }^{5,6}$ auftreten, da die Konstitution dieser Modelle sowohl im Hinblick auf den Aufbau ihrer Peptidkette als

1 22. Mitt.: „Über Fermentmodelle“ von W. Lautsch u. Mitarb.; 21. Mitt.: W. L a u t s c h, W. Ge hrmann, R. P a s edag u. Prater, Chem. Ber., im Druck; 20. Mitt.: W. L a u t s c h u. H. J. Kra e ge, Chem. Ber. 89, 737 [1956].

2 Vgl. Th. B ü c h e r, Angew. Chem. 62, 256 [1950].

3 O. Wa r b u r g, Schwermetalle als Wirkungsgruppen von Fermenten, Berlin 1948, S. 129.

4 P. Fröhlich u. H. M is chung, Kolloid-Z. 108, 30 [1944]; L. G o m b a y, Acta Univ. Szegediensis auch ihrer Wirkungsgruppe genau bekannt ist, was für die natürlichen Eiweißstoffe nur bedingt der Fall ist.

Wir zogen zu diesem Zweck die photochemische Spaltung der Kohlenoxyd-Verbindung der Mesohämin-IX-poly-DL-(phenylalanin-glutaminsäure) heran (vgl. Formel I a).

\section{Einstrahlung in die Banden der Wirkungsgruppen}

Als erstes galt es, festzustellen, mit welcher Quantenausbeute diese Reaktion bei Einstrahlung des spaltenden Lichtes in die Banden der Wirkungsgruppen verläuft. Hierzu bestrahlten wir in Anlehnung an die Methoden von $\mathrm{B}$ ü cher und Negele in ${ }^{7}$, die sich in einem Trog befindende Lösung der Kohlenoxyd-Verbindung des Fermentmodells, dessen Mol.-Gew. durch Spektrenvergleich zu 17800 ermittelt wurde, mittels einer besonderen Beleuchtungsoptik intermittierend mit monochromatischem Spaltungslicht (vgl. Abb. 1) der Wellenlänge $405 \mathrm{~m} \mu$. Senkrecht hierzu wurde der Trog von Meßlicht der Wellenlänge $546 \mathrm{~m} \mu$ durchsetzt, bei der sich die Absorption des Hämochromogens von der der CO-Verbindung besonders stark unterscheidet (vgl. Abb. 2). Die Intensität des Meßlichtes hinter dem Trog wurde von einer CdS-Zelle gemessen und nach elektronischer Verstärkung von einem Linienschreiber in Ab-

Sect. Sci. natur., Acta chem. physica 2, 196 [1949]; 241 [1950], zitiert nach C. A. 1950, $6257 \mathrm{e} ; 1$ 1951, 8359 h.

5 W. L a ut s c h u. E. S c h röd e r, Z. Naturforschg. 9 b, 277 [1954].

6 W. Lauts ch, H. Gnichtel, I. Gnichtel u. E. H ö fling, Kolloid-Z. 141, 132 [1955]; vgl. auch W. L a u t s c h u. Mitarbb., Kolloid-Z. 144, 82 [1955].

7 O. W a rburg u. E. N e ge le in, Biochem. Z. 200, 414 [1928]; 202, 202 [1929]; 204, 495 [1928]; Th. B ü cher u. E. Negelein, Biochem. Z. 311, 163 [1942]. 
hängigkeit von der Zeit registriert. Die Bestrahlungsstärke $E\left[\mathrm{~W} / \mathrm{cm}^{2}\right]$ des spaltenden Lichtes wurde mit einem Bolometer gemessen, das an ein Normal des NBS. angeschlossen war. Daraus wurde die Quantenintensität berechnet.

$$
\text { I }\left[\begin{array}{l}
\mathrm{Mol} \text { Phot } \\
\mathrm{cm}^{2} \cdot \min
\end{array}\right]
$$

Das Produkt $A=I \cdot a\left[\begin{array}{c}\text { Mol Phot } \\ \text { MolSubstanz } \cdot \min \end{array}\right]$ aus der Quantenintensität $I$ und dem leicht zu bestim-

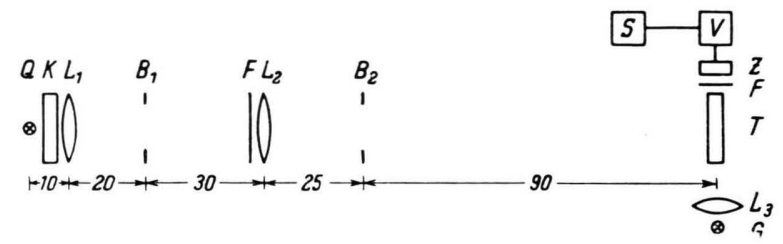

Abb. 1. Versuchsanordnung (schematisch). Q: Quecksilberlampe, K: Wasserküvette, B: Blenden, L: Linsen, F: Filter, G: Glühlampe, T: Meßtrog, Z: CdS-Zelle, V: Verstärker, S: Schreiber.

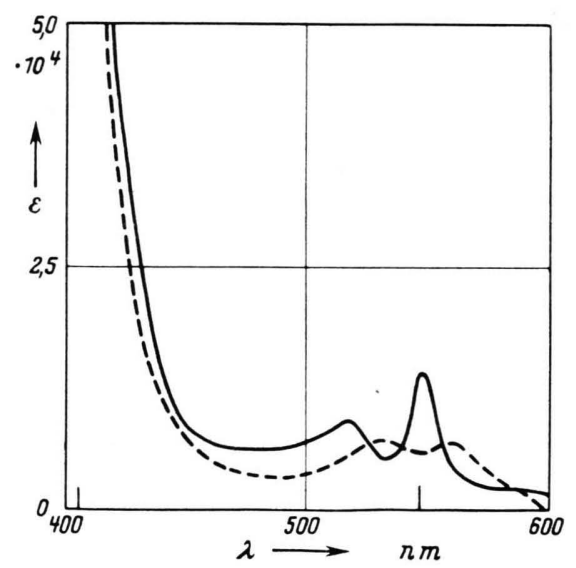

Abb. 2. Spektren des Hämochromogens (- $(-)$ und des Kohlenoxyd-Hämochromogens (- . -) der Mesohämin-IXpoly-DL-(phenylalanin-glutaminsäure). $c=2 \cdot 10^{-5} \mathrm{Mol} / l$.

menden Lichtabsorptions-Koeffizienten $a\left[\mathrm{~cm}^{2} / \mathrm{Mol}\right]$ ist die von einem Molekül der Kohlenoxyd-Verbindung je min absorbierte Anzahl von Photonen.

Zur Ermittlung der Quantenausbeute

$$
q=\frac{z_{\mathrm{i}}}{A}\left[\frac{\text { Mol Subst. }}{\text { Mol Phot }}\right]
$$

ist noch die photochemische Zerfallskonstante $z_{\mathrm{i}}\left[\mathrm{min}^{-1}\right]$ der Kohlenoxyd-Verbindung erforderlich. Da bei unseren Versuchen stets Hämochromogenbildner zugegen sind, liegt folgende Reaktion vor

$$
[\mathrm{Fe}] \mathrm{CO}+\mathrm{N} \backslash \underset{0}{\stackrel{\omega}{\rightleftarrows}}[\mathrm{Fe}] \mathrm{N} \frac{\mathrm{CO}}{\longleftarrow}
$$

Im Dunklen ist $\omega$ gleich der Dunkelzerfalls-Konstante, d. h. $\omega_{\mathrm{D}}=z_{\mathrm{D}}$; bei Bestrahlung tritt hierzu noch $z_{\mathrm{i}}$, d. h. $\omega_{\mathrm{H}}=\omega_{\mathrm{H}}+z_{\mathrm{i}} . \varrho$ ist die Geschwindigkeitskonstante der lichtunempfindlichen Rückreaktion. Im Dunklen ist das Gleichgewicht durch den Quotienten $\frac{\varrho}{\omega_{\mathrm{D}}}$, im Hellen durch den Quotienten $\frac{\varrho}{\omega_{\mathrm{D}}+z_{\mathrm{i}}}$ gegeben. Beim Belichten nach längerer Dunkelheit verschiebt sich das Gleichgewicht so lange nach rechts, bis das Hellgleichgewicht erreicht ist, bei Fortfall der Belichtung stellt sich wieder das Dunkelgleichgewicht ein. Für das Zeitgesetz dieser Vorgänge gilt

$$
\frac{\mathrm{d}([\mathrm{Fe}] \mathrm{CO})}{\mathrm{d} t}=-(\omega+\varrho) \cdot([\mathrm{Fe}] \mathrm{CO})+\varrho \geq[\mathrm{Fe}] .
$$

Da die Lichtabsorption der Kohlenoxyd-Verbindung von der des Hämochromogens verschieden ist, stellt die Änderung der Intensität des Meßlichtes hinter dem Trog ein Maß für den Umsatz dar. Wie eine nähere Rechnung zeigt, erhält man aus obiger Differentialgleichung

für die Hellperiode

$$
\begin{aligned}
& \left(\omega_{\mathrm{H}}+\varrho\right)=\frac{1}{t} \ln \frac{J_{\mathrm{D}}-J_{\mathrm{H}}}{J_{\mathrm{t}}-J_{\mathrm{H}}}, \\
& \left(\omega_{\mathrm{D}}+\varrho\right)=\frac{1}{t} \ln \frac{J_{\mathrm{H}}+J_{\mathrm{D}}}{J_{\mathrm{t}}+J_{\mathrm{D}}} .
\end{aligned}
$$

für die Dunkelperiode $\quad\left(\omega_{\mathrm{D}}+\varrho\right)=\frac{1}{t} \ln \frac{J_{\mathrm{H}}+J_{\mathrm{D}}}{J_{\mathrm{t}}+J_{\mathrm{D}}}$. $J_{\mathrm{D}}, J_{\mathrm{H}}, J_{\mathrm{t}}$ bedeuten dabei die für die Dunkel- bzw. Hellgleichgewichte sowie die zur Zeit $t$ charakteristischen Lichtintensitäten bzw. die entsprechenden Galvanometerausschläge. Diese Beziehungen stellen eine spezielle Form der 1928 von $\mathrm{W}$ a r b u r g ${ }^{7}$ abgeleiteten Gleichungen dar. Da alle J-Werte der Messung zugänglich sind, kann $\left(\omega_{\mathrm{H}}+\varrho\right)$ sowie $\left(\omega_{\mathrm{D}}+\varrho\right)$ und aus deren Differenzen $z_{\mathrm{i}}$ ermittelt werden.

In Abb. 3 ist als Beispiel eine Registrierkurve wiedergegeben. Die Auswertung dieser Kurven nach den obigen Gleichungen führte zu den Werten

$$
\begin{aligned}
\left(\omega_{\mathrm{H}}+\varrho\right) & =9,49 \mathrm{~min}^{-1}, \\
\left(\omega_{\mathrm{D}}+\varrho\right) & =7,80 \mathrm{~min}^{-1}, \\
z_{\mathrm{i}} & =1,69 \mathrm{~min}^{-1} .
\end{aligned}
$$

Die bolometrische Messung des spaltenden Lichtes ergab die Quantenintensität

$$
I_{\mathrm{eff}}=1,52 \cdot 10-8\left[\frac{\mathrm{Mol} \text { Phot }}{\mathrm{cm}^{2} \cdot \mathrm{min}}\right],
$$

der dekadische Extinktionskoffizient wurde zu $\varepsilon_{405}=$ $0,51 \cdot 10^{5} \mathrm{l} / \mathrm{Mol}$. cm gemessen, der natürliche Extinktionkoeffizient beträgt daher $a_{405}=2,3 \cdot 0,51 \cdot 10^{8}=$ $1,18 \cdot 10^{8} \mathrm{~cm}^{2} /$ Mol. Somit ergibt sich für die Quantenausbeute

$$
q=0,945\left[\begin{array}{c}
\mathrm{Mol} \mathrm{CO} \\
\text { Mol Phot }
\end{array}\right] .
$$

In gleicher Weise wurde auch die Spaltung der Kohlenoxyd-Verbindung von Cytohämin-poly-L-(phe- 
nylalanin-glutaminsäure) (vgl. Formel I b) gemessen, deren Mol.-Gew. durch Spektrenvergleich zu 9100 ermittelt wurde und deren Spektren Abb. 4 zeigt. Die Konzentration der Meßlösung betrug 9,9 $10^{-6}$ $\mathrm{Mol} / l$, als Lösungsmittel diente eine wäßrige $0,5-m$. Imidazollösung, die mit Kohlenoxyd gesättigt und mit Natriumdithionit reduziert wurde. Der Lichtabsorptions-Koeffizient betrug $a_{405}=1,06 \cdot 10^{8} \mathrm{~cm}^{2} /$ Mol, die Quantenintensität wurde zu $I_{\text {eff }}=1,15 \cdot 10^{-8}$

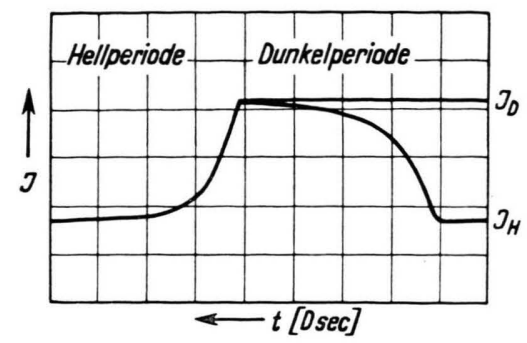

Abb. 3. Registrierkurve der Kohlenoxydspaltung von Mesohämin-IX-poly-DL-(phenylalanin-glutaminsäure).

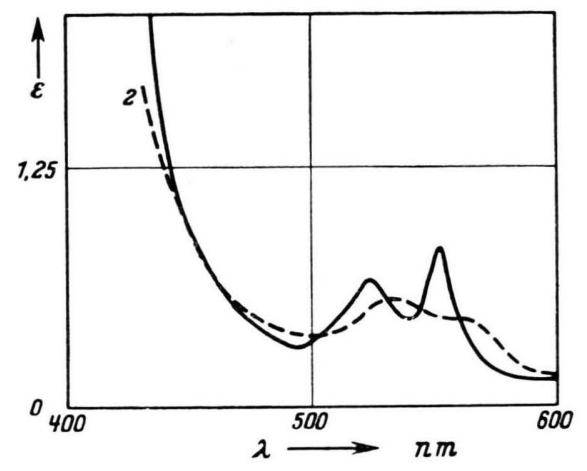

Abb. 4. Spektren des Hämochromogens (- und des Kohlenoxyd-Hämochromogens (----) der Cytohämin-polyL-(phenylalanin-glutaminsäure). $\quad c_{1}=1,74 \cdot 10^{-5}, \quad c_{2}=$ $1,62 \cdot 10^{-5}$.

$\left[\frac{\mathrm{Mol} \text { Phot }}{\mathrm{cm}^{2} \cdot \mathrm{min}}\right]$ und die photochemische Zerfallskonstante zu $z_{\mathrm{i}}=1,2 \mathrm{~min}^{-1}$ ermittelt. Daraus ergibt sich die Quantenausbeute

$$
q=0,985\left[\begin{array}{c}
\text { Mol CO } \\
\text { Mol Phot }
\end{array}\right] .
$$

In beiden Fällen wurde also für die photochemische Spaltung der Kohlenoxyd-Verbindung unserer Fermentmodelle innerhalb der Meßgenauigkeit die Quantenausbeute eins erhalten.

\section{Einstrahlung in die Bande des Phenylalanins}

Nachdem auf diese Weise gezeigt war, daß sich unsere Modelle in dieser Hinsicht genau so verhalten wie die anderen Kohlenoxyd-Eisenverbindungen, führten wir die Spaltung an Mesohämin IX-peptid

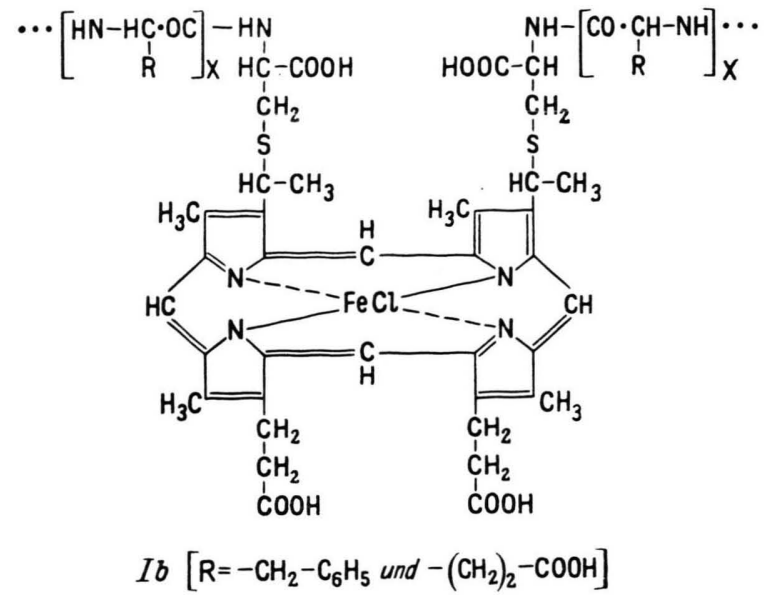

mit Licht der Wellenlänge $254 \mathrm{~m} \mu$ durch, das, wie Abb. 5 zeigt, nicht nur von der Wirkungsgruppe, sondern auch von den Phenylalaninresten der Peptidkette absorbiert wird. Hierzu konnte als Hämochromogen-Bildner nicht Pyridin Verwendung finden, da es in diesem Spektralgebiet zu stark absorbiert; wir wendeten deshalb Imidazol an, das eine hinreichend kleine Absorption besitzt $\left(\varepsilon_{254}=0,14\left[\begin{array}{c}l \\ \mathrm{Mol} \cdot \mathrm{cm}\end{array}\right]\right.$ gegenüber $\varepsilon_{254}=0,15 \cdot 10^{5}\left[\frac{l}{\mathrm{Mol} \cdot \mathrm{cm}}\right]$ des Kohlenoxydhämochromogens).

Um jedoch etwaige, durch die Absorption des Hämochromogen-Bildners bedingte Fehler auszuschalten - zumal bei diesem Versuch die Monochromasie des spaltenden Lichtes nicht sonderlich gut war - , wurde nach einem Relativverfahren gearbeitet, indem unter gleichen Bedingungen sowohl der freien Wirkungsgruppe, d. h. des Mesohämins IX, als auch die Zerfallskonstante $z_{i}^{\text {W }}$ der Kohlenoxydverbindung des Modells bestimmt wurde. Aus dem Verhältnis der Zerfallskonstanten

$$
\frac{z_{\mathrm{i}}^{\mathrm{W}}}{z_{\mathrm{i}}^{\mathrm{M}}}=\frac{\varphi^{\mathrm{W}} \cdot a^{\mathrm{W}}}{\varphi^{\mathrm{M}} \cdot a^{\mathrm{M}}}
$$

folgt mit

$$
\begin{aligned}
& q^{\mathrm{W}} \equiv 1 \\
& \tau^{\mathrm{M}}=\begin{array}{l}
z_{\mathrm{i}}^{\mathrm{H}} \cdot a^{\mathrm{W}} \\
z_{\mathrm{i}}^{\mathrm{W}} \cdot a^{\mathrm{M}} .
\end{array}
\end{aligned}
$$

Mit den experimentell erhaltenen Werten

$$
\begin{array}{cl}
z_{\mathrm{i}}^{\mathrm{W}}=1,30 \mathrm{~min}^{-1} ; & z_{\mathrm{i}}^{\mathrm{M}}=2,00 \mathrm{~min}^{-1} ; \\
a_{254}^{\mathrm{W}}=3,45 \cdot 10^{\tau} \mathrm{cm}^{2} / \mathrm{Mol} ; & a_{254}^{\mathrm{M}}=5,98 \cdot 10^{\tau} \mathrm{cm}^{2} / \mathrm{Mol}
\end{array}
$$

ergibt sich für die Quantenausbeute des Modells

$$
\tau^{\mathrm{M}}=0,89\left[\frac{\mathrm{Mol} \mathrm{CO}}{\text { MolPhot }}\right] \text {. }
$$


Aus diesem Wert, dessen Abweichung von eins innerhalb der bei diesem Versuch beträchtlichen Fehlergrenze liegt, sowie allein schon aus der Tatsache, daß $z_{i}{ }^{\mathrm{M}}>z_{i}$ ", ergibt sich, daß auch die von der Peptidkette absorbierten Photonen zumindest teilweise an der prosthetischen Gruppe wirksam werden. Es muß also ein Energietransport von der Peptidkette zum Eisen der Wirkungsgruppe erfolgt sein. Nähere Aussagen über die Art dieses Energietransportes können indessen erst gemacht werden, wenn die Ergebnisse der z. Z. anlaufenden genaueren Untersuchungen vorliegen.

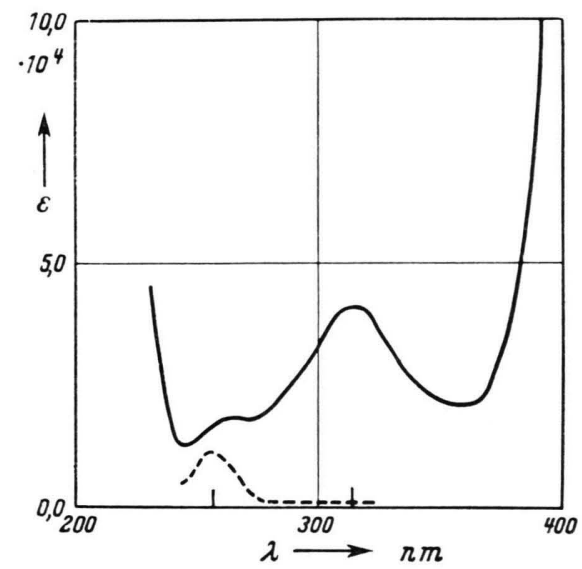

Abb. 5. UV-Spektren. (— Kohlenoxyd-Hämochromogen des Mesohämin IX. (- - -) Poly-DL-(phenylalaninglutaminsäure $1: 1$ ), berechnet auf $M=17800$.

Es erscheint aber schon jetzt sinnvoll, die verschiedenen Möglichkeiten zu diskutieren.

\section{Diskussion}

Für die Energiefortleitung in Eiweißstoffen nahm S zent-György i ${ }^{8}$ bereits 1941 an, daß — ähnlich wie beim Bändermodell des Festkörpers - die „äußeren“ Elektronen des Eiweißteilchens ein sich über das ganze Teilchen erstreckendes gemeinsames Elektronensystem bilden, bei denen die Energieterme der Einzelelektronen zu Bändern verschmolzen sind, die sich über eine große Zahl von Teilchen erstrekken und nicht auf die einzelne Mizelle begrenzt sind.

Andere Autoren schreiben die Leitfähigkeit nicht so summarisch dem ganzen Eiweißteilchen zu, sondern der in ihm enthaltenen charakteristischen Atomgruppierung, der Peptidgruppe.

Nach $\mathrm{W}$ ir tz ${ }^{9}$ erfolgt die Verschiebung einer elektri-

8 A. Szent-Györgyi, Science [New York] 93, 609 [1941]; Nature [London] 157, 875 [1946].

9 K. W i r t z, Z. Naturforschg. 2 b, 94 [1947].

10 W. Schmitt u. R. Purrmann, Z. Natur- schen Ladungseinheit über ein System kooperierender durch Protonbrücken verknüpfter Peptidgruppen im Zuge einer Laktam-Laktim-Tautomerie. Charakteristisch für diese Vorstellung ist, daß über ein derartiges System nur eine einzige Elementarladung verschoben werden kann und daß zur Regenerierung des Systems, d. h. zur Rückbildung der Lactamform, ein Rücktransport dieser Ladung erfolgen muß. Eine kontinuierliche Leitfähigkeit ist also nach diesem Mechanismus nicht gegeben.
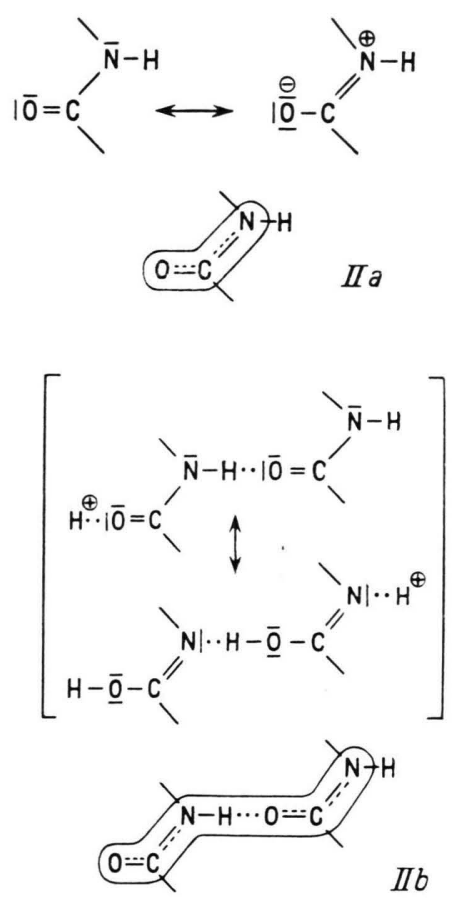

Diese Schwierigkeit vermeidet die "Amidkettentheorie“ von $\mathrm{Schmitt}$ und $\mathrm{Pur}$ rmann ${ }^{10}$, nach der die Leitfähigkeit dadurch zustande kommt, daß benachbarte Peptidgruppen ein „elektroduktiles System“ bilden. Dabei soll ein Elektronenübergang zwischen den einzelnien, durch Wasserstoffbrücken zusammengehaltenen Peptidgruppen benachbarter Peptidketten - beispielsweise in einer $\beta$-Keratinstruktur - möglich sein, indem die $\pi$ Elektronenwolken (vgl. Formel II a) der einzelnen mesomeren Peptidgruppen miteinander verschmelzen oder indem — anders ausgedrückt — es zu einer Überlappung ihrer Amplitudenfunktion kommt 11 (vgl. Formel II b). Dadurch bilden sich quer zur Richtung der Polypeptidkette „Leitfähigkeitsbänder“ aus, über die ein ElektronenTransport möglich ist. Nach dieser Vorstellung können also in beliebiger Richtung beliebig viele Elektronen quer zur Richtung der Peptidketten transportiert werden, ohne daß eine stoffliche Veränderung (z. B. Verschiebung eines Tautomerie-Gleichgewichtes) erfolgt. Diese Ansicht stellt somit ein etwas detailliertes Bild der S z e n t-G y ör g y i schen Vorstellung dar.

forschg. 3 b, 411 [1948].

11 M. G. Evans u. J. Gergeley, Biochim. biophysica Acta [Amsterdam] 3, 188 [1949]. 
Während bei den bisher angeführten Mechanismen der Energietransport mit einer Verschiebung elektrischer Ladungen (Ionen oder Elektronen) verknüpft ist, nehmen $\mathrm{Scheibe}{ }^{12}$ und $\mathrm{F}$ örster ${ }^{13}$ die gegenseitige elektrodynamische Kopplung zwischen den Elektronensystemen der beteiligten gleichartigen oder nahezu gleichartigen Moleküle - im vorliegenden Fall wohl zwischen denen der benachbarten Peptidgruppen - als Ursache des Energieübergangs an, die von keinerlei Übergang oder Austausch von Elektronen begleitet ist.

Zum Verständnis einer Leitfähigkeit längs der Peptidkette kann man auf folgenden zwei Wegen gelangen.

Man kann erstens davon ausgehen, daß es sich bei den in den natürlichen Eiweißstoffen vorkommenden Aminosäuren stets um $\alpha$-Aminosäuren mit einem $\alpha$ ständigen H-Atom handelt. Damit

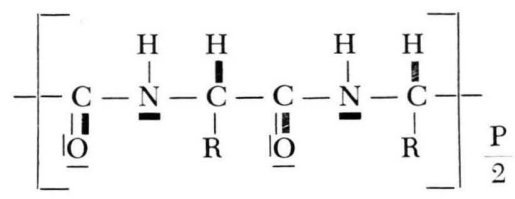

ist Überkonjugation gegeben; d. h. das bindende Elektronenpaar der $\mathrm{C}-\mathrm{H}$-Bindung kann mit der Doppelbindung der $\mathrm{C}=\mathrm{O}$-Bindung in eine ähnliche Wechselwirkung treten, wie diese mit einer zweiten Doppelbindung oder mit einem einsamen Elektronenpaar, die $\mathrm{C}-\mathrm{H}$-Bindung ist also ähnlich wie ein einsames Elektronenpaar „konjugationsfähig“. Ein Blick auf obige Formel zeigt nun, daß jedes Atom der Peptidkette eine konjugationsfähige Bindung oder ein einsames Elektronenpaar besitzt, so daß die Ausbildung eines „konjugierten Systems“, d.h. eines Elektronengases, mithin auch eine Leitfähigkeit längs der Peptidkette möglich erscheint. Nach dieser Vorstellung sollten Polypeptide aus $\beta$-Aminosäuren, mit deren Aufbau wir z. Z. beschäftigt sind, keine Energieleitung zeigen.

Während obiger Mechanismus unabhängig von der räumlichen Struktur des Peptidmoleküls ist, ist die folgende zweite Vorstellung über die Leitfähigkeit entlang eines Peptidmoleküls an das Vorliegen einer gewendelten Anordung, wie sie von $\mathrm{Pauling}{ }^{14}$ z. B. für das $\alpha$-Keratin angenommen wird, gebunden. In diesem Fall steht in der Wendel eine Peptidgruppe mit der in derselben Peptidkette folgenden

12. G. Scheibe, Z. Elektrochem. angew. physik. Chem. 52, 283 [1948].

13 Th. Förster, Ann. Physik (6) 2, 55 [1948]. dritten Gruppe in einer räumlichen Beziehung, wie sie weiter oben für Gruppen benachbarter Peptidketten bei der $\beta$-Struktur angeschrieben wurde. Dies hat zur Folge, daß sich längs einer Wendel drei parallele Bahnen von einander benachbarten Peptidgruppen hinziehen, über die eine Energiefortleitung nach einem der oben diskutierten Mechanismen erfolgen kann (vgl Abb.6). Diejenigen Mechanismen, die bei der $\beta$-Struktur zu einer Energiefortleitung quer zur Peptidkette führen, verursachen also bei der $a$-Struktur eine Fortleitung längs der Wendel und damit längs der Peptidkette.

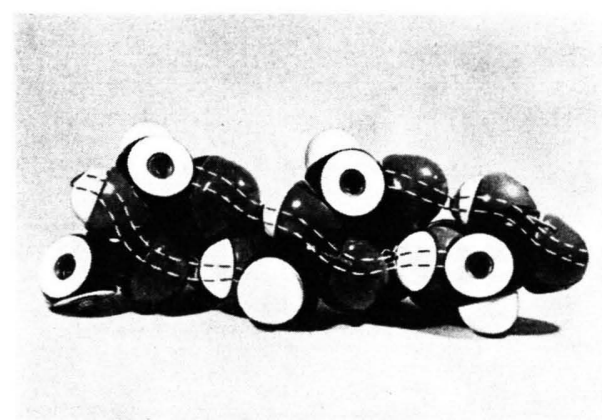

Abb. 6. Modell einer Polypeptidkette nach Pauling mit eingezeichneten „Leitfähigkeitsbahnen“, die über benachbarte Peptidgruppen laufen.

Beim Vorliegen einer Leitfähigkeit längs der Peptidwendel ist noch mit einem weiteren Phänomen zu rechnen. Da die Leitfähigkeit über die Peptidgruppen von deren genügender räumlicher Annäherung abhängt, so muß jede Veränderung der "Ganghöhe“ der Wendel Rückwirkungen auf die Energiefortleitung haben. Je kleiner die „Ganghöhe“, d. h. je stärker die Annäherung der Peptidgruppen, desto gröBer wird die Leitfähigkeit sein und umgekehrt.

Nun ist aber die „Ganghöhe" der Wendel nicht konstant, da die einzelnen „Gänge“ der Wendel infolge der Temperaturbewegungen Schwingungen gegeneinander ausführen. Die Leitfähigkeit wird also eine Funktion der Temperatur sein. Weiterhin muß die Leitfähigkeit durch spezifische Anregung der Schwingung der Wendel gegeneinander etwa mit infrarotem Licht, in dessen Bereich vermutlich die Eigenfrequenz der Wendel fallen dürfte, beeinflußbar sein. Nähere Aussagen hierüber können indessen erst nach eingehenderen theoretischen und experimentellen Untersuchungen gemacht werden.

14 L. P a uling, Proc. nat. Acad. Sci. USA 37, 205 [1951]. 
Während vorstehend nur der Transport von elektrischen Ladungen diskutiert wurde, bietet die gewendelte Peptidkette darüber hinaus noch die Möglichkeit, kinetische Energie ohne allzu große Verluste gerichtet (und zwar längs der Wendel) weiterzuleiten. Regt man eine derartige Wendel etwa durch einen Stoß an einem Ende zu Schwingungen längs der Wendel an, so pflanzt sich diese Schwingung, wie man am Modell einer Federwendel leicht zeigen kann, bis zum anderen Ende fort.

Zum Schluß soll noch die Frage nach dem Energieübergang von der Peptidkette zur prosthetischen Gruppe ganz kurz erörtert werden. Im Falle der zuletzt erwähnten Übertragung von Bewegungsenergie ist dieser Übergang ohne weiteres gegeben. Im Falle der Elektronenleitung tritt eine gewisse Schwierigkeit insofern auf, als die „Konjugation“ zwischen der

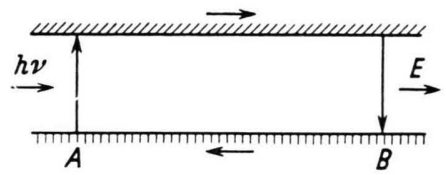

Abb. 7. Schema eines Energietransportes durch Elektronenleitung. A: Ort der Energieaufnahme durch Photonenabsorption. B: Ort der Energieabgabe durch Rückkehr des Elektrons auf das untere Niveau. Zwischen A und B erstreckt sich die Peptidkette.

Peptidkette und der prosthetischen Gruppe durch die Methylengruppen der Propionsäurereste unterbrochen ist. Man kann daran denken, daß das Elektron, das durch das von dem Phenlyalaninrest (Stelle A in Abb. 7) absorbierte Photon auf ein höheres Niveau gehoben wurde, dort über die Peptidgruppen in Richtung auf die Wirkungsgruppe „wandert“ und innerhalb des dieser benachbarten Peptidkettenteils (Stelle $B$ in Abb.7) unter Energieabgabe auf das untere Niveau herabfällt und in ihm über die Peptidgruppen zum Phenylalaninrest zurückkehrt. Die in der Nähe des Eisens frei gewordene Energie führt dann zur Spaltung der Kohlenoxyd-Verbindung. Möglicherweise "gelangt" das Elektron aber auch über das Imidazol ${ }^{10}$ bis

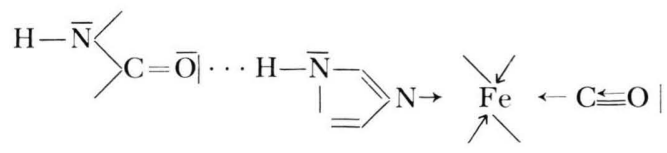

zur prosthetischen Gruppe, die in diesem Fall durch $\mathrm{B}$ in Abb. 7 angedeutet wird, und vollzieht dort den Übergang auf die tiefere Bahn. Wie Abb. 8 zeigt, ist eine derartige Anordnung räumlich durchaus mög- lich. Für diesen Mechanismus, der ein neues Licht auf die Rolle der Hämochromogen-Bildner wirft, spricht, daß die Spaltung bisher nur in ihrer Gegenwart beobachtet wurde.

Zur Prüfung, ob die hier skizzierten neuen Vorstellungen der Tatsache entsprechen, dürften jedoch noch eine ganze Anzahl gründlicher Untersuchungen erforderlich sein. Besonders aussichtsreich erscheint es uns, unsere relativ einfachen Modelle zu verwenden. Wir sind deshalb z. Z. damit beschäftigt, nicht nur

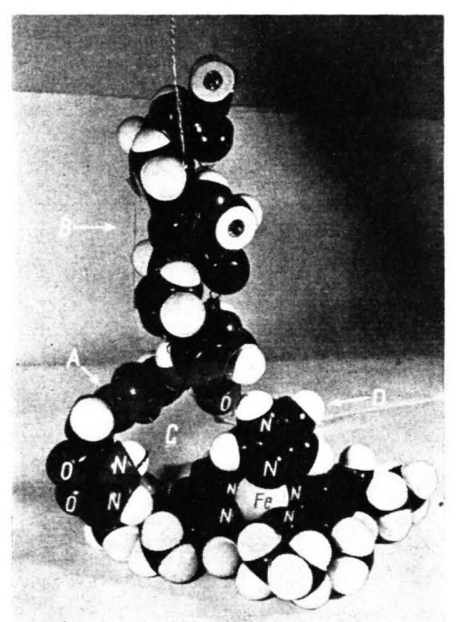

Abb. 8. Modell des Imidazol-Hämochromogens des Mesohämin-Polypeptids. Die Aminosäurereste sind der besseren Übersichtlichkeit halber weggelassen. Auf der anderen Seite der Mesohämin-Scheibe hat man sich eine entsprechende Anordnung zu denken. A: Offene Peptidkette, B: Peptidwendel, C: Raum für einen Aminosäurerest, D: Imidazol. Man erkennt, daß das O-Atom der letzten Peptidgruppe der Wendel durch eine H-Brücke mit dem Imidazol, das seinerseits mit dem $\mathrm{Fe}$ verbunden ist, verknüpft ist.

die Untersuchung der photochemischen Spaltung der CO-Verbindungen unserer Modelle im UV mit genaueren Methoden und unter wechselnden Versuchsbedingungen zu wiederholen, sondern beginnen auch mit Versuchen, die klären sollen, welchen Einfluß die Einwirkung von Infrarotstrahlen, die die einzelnen Wendelgänge zu Schwingungen gegeneinander anregen, auf Energieleitungs-Vorgänge haben.

Ferner erwarten wir, daß die Frage, ob eine Übertragung mechanischer Energie durch die Wendel möglich ist, durch die anlaufende eingehende kinetische Untersuchung der Dunkelreaktionen der COVerbindungen unserer Modelle geklärt werden kann.

\section{Versuchsteil}

A. Die verwendeten Fermentmodelle wurden nach den bereits früher mitgeteilten Verfahren hergestellt 5, 6 . 
B. Die Mol.-Gew. der Modelle wurden durch Vergleich der Spektren ihrer Hämochromogene mit denen der entsprechenden freien Wirkungsgruppe ermittelt.

1. Mesohämin-IX-poly-DL-(phenylalanin-glutaminsäure $1: 1)$

a) Vergleichslösung: $1,64 \mathrm{mg}=2,5 \cdot 10^{-6} \mathrm{Mol}$ Mesohämin-IX werden in $3,3 \mathrm{ml}$ Wasser und $3,3 \mathrm{ml}$ Boratpuffer $p_{\mathrm{H}} 9,45$ gelöst und mit Pyridin auf $10 \mathrm{ml}$ aufgefüllt $\left(c=2,5 \cdot 10^{-4} \mathrm{Mol} / l\right) .2 \mathrm{ml}$ dieser Lösung werden mit $12 \mathrm{ml}$ Pyridin und $13 \mathrm{ml}$ Boratpuffer verdünnt, durch Durchleiten eines $\mathrm{O}_{2}$-freien $\mathrm{N}_{2}$-Stromes luftfrei gemacht; dann wird mit 0,01-m. Cysteinhydrochloridlösung auf $50 \mathrm{ml}$ aufgefüllt $\left(c=1 \cdot 10^{-5} \mathrm{Mol} / l\right)$. Die ExtinktionsKoeffizienten dieser Lösung wurden bei 517 und $647 \mathrm{~m} \mu$ zu

$$
\varepsilon_{517}=1,12 \cdot 10^{4} \text { bzw. } \varepsilon_{547}=1,60 \cdot 10^{4}\left[\begin{array}{c}
l \\
\mathrm{Mol} \cdot \mathrm{cm}
\end{array}\right]
$$

gemessen.

b) Modellösung: 60,1 mg Mesohämin-IX-poly-DL-(phenylalanin-glutaminsäure) werden in $6,5 \mathrm{ml}$ Boratpuffer und $6,5 \mathrm{ml}$ Pyridin gelöst. Nach Durchleiten von $\mathrm{N}_{2}$ wird diese Lösung mit 0,01-m. Cysteinhydrochloridlösung auf $25 \mathrm{ml}$ aufgefüllt $(c=2,4 \mathrm{~g} / l)$. Die Extinktionen bei einer Schichtdicke von $0,5 \mathrm{~cm}$ wurden $\mathrm{zu}$

$$
E_{517}=0,738 \text { bzw. } E_{547}=1,103
$$

gemessen.

c) Die Wirkungsgruppen-Konzentration ergibt sich daraus zu:

$$
c=\frac{0,738}{1,12 \cdot 0,5} \cdot 10^{-4}=1,32 \cdot 10^{-4}
$$

bzw.

$$
\begin{aligned}
c= & \frac{1,103}{1,60 \cdot 0,5} \cdot 10^{-4}=1,38 \cdot 10^{-4} \\
& \text { Mittelwert } c=1,35 \cdot 10^{-4} \mathrm{Mol} / l .
\end{aligned}
$$

Für den Fall, daß jedes Polypeptid-Molekül eine Wirkungsgruppe als Endgruppe besitzt, folgt

$$
1,35 \cdot 10^{-4} \mathrm{Mol}=2,4 \mathrm{~g},
$$

d. h. das Mol.-Gew. (Zahlendurchschnitt) beträgt 17800 .

2. Cytohämin-poly-L-(phenylalanin-glutaminsäure $1: 1)$

a) Modellösung: 7,9 mg Modell werden in $50 \mathrm{ml} \mathrm{0,5-m}$. wäßriger Imidazollösung gelöst und nach Vertreiben der Luft durch Durchleiten von $\mathrm{N}_{2}$ mit einigen Körnchen $\mathrm{Na}_{2} \mathrm{~S}_{2} \mathrm{O}_{4}$ reduziert $(c=0,158 \mathrm{~g} / l)$. Die Extinktionen bei 521 und $551 \mathrm{~m} \mu$ wurden bei einer Schichtdicke von $1 \mathrm{~cm}$ $\mathrm{zu}$

ermittelt.

$$
E_{521}=0,118 \text { bzw. } E_{551}=0,145
$$

b) Die Wirkungsgruppen-Konzentration ergibt sich hieraus und aus den Extinktions-Koeffizienten

$$
\varepsilon_{521}=0,06 \cdot 10^{5} \text { bzw. } \varepsilon_{551}=0,096 \cdot 10^{5}\left[\frac{l}{\mathrm{Mol} \cdot \mathrm{cm}}\right]
$$

die an einer analog der Modellösung hergestellten Lösung des freien Cytohämins $\left(c=10^{-5} \mathrm{Mol} / l\right)$ gemessen wurden, zu

$$
\begin{gathered}
c=\frac{0,118}{0,06} \cdot 10^{-5}=1,97 \cdot 10^{-5} \\
\text { bzw. } c=\frac{0,145}{0,096} \cdot 10^{-5}=1,51 \cdot 10^{-5} \mathrm{Mol} / l . \\
\text { Mittelwert : } c=1,74 \cdot 10^{-5} \mathrm{Mol} / \mathrm{l} .
\end{gathered}
$$

Daraus folgt:

$$
\begin{aligned}
1,74 \cdot 10^{-5} \mathrm{Mol} & =1,58 \cdot 10^{-1} \mathrm{~g}, \\
1 \mathrm{Mol} & =9,1 \cdot 10^{3} \mathrm{~g},
\end{aligned}
$$

d. h. das Mol.-Gew. (Zahlendurchschnitt) beträgt 9100.

C. Messung der Spektren

a) CO-Verbindung des Mesohämins IX im UV: $1,64 \mathrm{mg}$ $=2,5 \cdot 10^{-6} \mathrm{Mol}$ Mesohämin IX werden in $3,3 \mathrm{ml}$ Boratpuffer $p_{\mathrm{H}} 9,45$ gelöst und mit Wasser auf $10 \mathrm{ml}$ aufgefüllt $\left(c=2,5 \cdot 10^{-4} \mathrm{Mol} / l\right) .1 \mathrm{ml}$ dieser Lösung wird mit $n / 10$-Natronlauge auf $50 \mathrm{ml}$ aufgefüllt und nach Durchleiten von $\mathrm{CO}$ durch Zusatz von einigen Körnchen fester Glucose reduziert $\left(c=5 \cdot 10^{-6} \mathrm{Mol} / l\right)$. Das Spektrum dieser Lösung wurde gemessen (vgl. Abb. 5).

$\beta$ ) Hämochromogen der Mesohämin IX-poly-DL-(phenylalanin-glutaminsäure). $44,5 \mathrm{mg}=2,5 \cdot 10^{-6} \mathrm{Mol} \mathrm{Mo-}$ dell werden in $5 \mathrm{ml}$ Pyridin und $5 \mathrm{ml}$ Boratpuffer $p_{\mathrm{H}} 9,45$ gelöst $\left(c=2,5 \cdot 10^{-4} \mathrm{Mol} / l\right) .4 \mathrm{ml}$ dieser Lösung werden mit Pyridin-Pufferlösung (1:1) auf $25 \mathrm{ml}$ aufgefüllt. Nach Vertreiben der Luft durch Durchleiten eines Stickstoffstromes wird durch Zugabe von $25 \mathrm{ml}$ einer 0,01-m. Cysteinhydrochloridlösung reduziert $\left(c=2 \cdot 10^{-5} \mathrm{Mol} / l\right)$. Das Spektrum dieser Lösung wurde gemessen (vgl. Abb. 2).

$\gamma)$ Kohlenoxyd-Hämochromogen des Mesohämin-Modells. Durch die obige Lösung wurde $10 \mathrm{~min}$ lang ein CO-Strom geleitet, dann wurde das Spektrum aufgenommen (vgl. Abb. 2).

ঠ) Hämochromogen der Cytohämin-poly-L-phenyl-alanin-glutaminsäure). Es wurde die unter 2 a beschriebene Lösung verwendet $\left(c=1,74 \cdot 10^{-5} \mathrm{Mol} / l\right)$ (vgl. Abb. 4).

$\varepsilon)$ Kohlenoxydhämochromogen des Cytohämin-Modells. In die nach den unter 2 a gemachten Angaben hergestellte Lösung, bei der jedoch von 7,4 mg Modell ausgegangen wurde, wurde $10 \mathrm{~min}$ lang ein $\mathrm{CO}$-Strom geleitet, dann wurde das Spektrum gemessen $\left(c=1,62 \cdot 10^{-5} \mathrm{Mol} / l\right)(\mathrm{vgl}$. Abb. 4).

D. Messung der photochemischen Spaltung durch Licht der Wellenlänge $405 \mathrm{~m} \mu$

1. $17,8 \mathrm{mg}=10^{-6} \mathrm{Mol}$ Mesohämin-Modell werden in $12 \mathrm{ml}$ Pyridin und $13 \mathrm{ml}$ Boratpuffer $p_{\mathrm{H}} 9,45$ gelöst $\left(c=4 \cdot 10^{-5} \mathrm{Mol} / l\right)$. $2,5 \mathrm{ml}$ dieser Lösung werden mit 22,5 ml Pyridin-Pufferlösung (1:1) verdünnt; im Eisbade wird Stickstoff durchgeleitet, dann wird mit $25 \mathrm{ml} \mathrm{0,01-m}$. Cysteinhydrochloridlösung reduziert, worauf, immer im Eisbad, 15 min lang ein Gasstrom $\left(80 \% \mathrm{~N}_{2}+20 \% \mathrm{CO}\right)$ durchgeleitet wird. Diese Lösung $\left(c=2 \cdot 10^{-6} \mathrm{Mol} / l\right)$ wurde in die Meßküvette $(10 \cdot 1 \cdot 2 \mathrm{~cm})$ gedrückt und intermittierend mit Spaltlicht (Höchstdrucklampe HBO 500, Beleuchtungsoptik lt. Abb. 1, Interferenzfilter $\lambda=405 \mathrm{~m} \mu$ ) bestrahlt. 
2. $9,0 \mathrm{mg}=9,9 \cdot 10^{-7} \mathrm{Mol}$ Cytohämin-Modell wird in $10 \mathrm{ml} 0,1-n$. Natronlauge gelöst $\left(c=9,9 \cdot 10^{-5} \mathrm{Mol} / l\right)$. $5 \mathrm{ml}$ davon werden mit $0,5-\mathrm{m}$. Imidazollösung auf $50 \mathrm{ml}$ verdünnt $\left(c=9,9 \cdot 10^{-6} \mathrm{Mol} / l\right)$. Nach Sättigen mit CO wird mit $\mathrm{Na}_{2} \mathrm{~S}_{2} \mathrm{O}_{4}$ reduziert. Diese Lösung wurde in die Meßküvette gedrückt und mit Spaltlicht $405 \mathrm{~m} \mu$ bestrahlt.

E. Messung der photochemischen Spalt ung durch U V-Lich t

$1,64 \mathrm{mg}$ Mesohämin-IX bzw. 44,5 mg Mesohämin-Modell $=2,5 \cdot 10^{-6} \mathrm{Mol}$ werden in $3,3 \mathrm{ml}$ Boratpuffer $p_{\mathrm{H}} 9,45$ gelöst und mit Wasser auf $10 \mathrm{ml}$ aufgefüllt. $1 \mathrm{ml}$ dieser Lösung wird mit $25 \mathrm{ml}$ Boratpuffer versetzt und nach Zugabe von 1,5 g Imidazol mit Wasser auf $50 \mathrm{ml}$ aufgefüllt. Nach Sättigen mit $5 \% \mathrm{CO}+95 \% \mathrm{~N}_{2}$ wird mit $\mathrm{Na}_{2} \mathrm{~S}_{2} \mathrm{O}_{4}$ reduziert. Diese Lösung $\left(c=5 \cdot 10^{-6} \mathrm{Mol} / l\right)$ wurde in die Meßküvette (Quarz) gedrückt und mit Spaltlicht [Quecksilberlampe, Beleuchtungsoptik, Flüssigkeitsfilter: $24,6 \mathrm{~g} \mathrm{NiSO}_{4} \cdot 7 \mathrm{H}_{2} \mathrm{O}+7 \mathrm{~g} \mathrm{CoSO} \cdot 7 \mathrm{H}_{2} \mathrm{O}$ in $100 \mathrm{ml}$ Wasser $(2 \mathrm{~cm})$ und $1,9 \mathrm{ml} \mathrm{CS}$, in $100 \mathrm{ml}$ Alkohol $(0,5 \mathrm{~cm})]$ bestrahlt. Zur Messung diente Licht der Wellenlänge $546 \mathrm{~m} \mu$ (Glühlampe, Interferenzfilter).

F. Allg e meins

Die Bestrahlungsstärke $E$ wurde mit einem Großflächenbolometer nach K u r l b a u m (Fa. H. Röhrig, Ber-
lin-Schöneberg) gemessen. Dieses wurde mittels einer Standardlampe des NBS. geeicht. Zwischen der Änderung $\vartheta[\Omega]$ des im Nebenschluß liegenden Widerstandes (der eigentlichen Meßgröße) und der Bestrahlungstärke $E$ (Watt $/ \mathrm{cm}^{2}$ ) wurde auf diese Weise die bis $E=10^{-3}$ Watt $/ \mathrm{cm}^{2}$ hinreichend genau geltende Beziehung

$$
-\vartheta=1,93 \cdot 10^{4} \cdot E
$$

ermittelt. Die Quantenintensität $I$ wurde aus der Bestrahlungsstärke $E$ nach der Formel

$$
I=5,024 \cdot 10^{-7} \cdot \lambda \cdot E
$$

berechnet (wobei $\lambda$ in $m \mu$ einzusetzen ist). Die in der Küvettenmitte herrschende (effektive) Quantenintensität ergibt sich daraus zu

$$
I_{\mathrm{eff}}=\frac{I}{2}(1+10-\varepsilon c) .
$$

Die Reaktionsgeschwindigkeits-Konstanten wurden entweder durch Analyse der Zeitkurven (D 1) oder aus den gemessenen Halbwertszeiten (D 2, E) errechnet.

Alle übrigen, hier nicht angeführten Zahlenwerte wurden bereits im Text mitgeteilt.

Wir danken Fräulein L e h m a n n und Fräulein S e i fert für ihre Mithilfe bei der Durchführung der Versuche.

\section{Konstitution und Wirkung biologisch aktiver Verbindungen}

\section{Mitt.: Diäthyl-thiophosphorsäure - $p$ - nitrophenylester (E 605) und Derivate}

\section{Von Werner Perkow}

Aus dem Forschungslaboratorium der Norddeutschen Affinerie, Hamburg

(Z. Naturforschg. 11 b, 460-463 [1956]; eingegangen am 9. April 1956)

Eine für die insektiziden Derivate der E 605-Gruppe charakteristische Elektronenverteilung wird für die Wechselwirkung mit Fermenten verantwortlich gemacht.

$\mathrm{B}$ ei Betrachtungen über die Zusammenhänge zwischen Konstitution und Wirkung des Di ( $p$-chlorphenyl)-trichloräthans (DDT) haben wir wahrscheinlich gemacht, daß eine charakteristische ElektronenKonfiguration mit der insektiziden Wirkung in $\mathrm{Zu}$ sammenhang steht. Die Substituenten-Verteilung aller wirksamen Verbindungen der DDT-Gruppe begünstigt eine Konfiguration, die durch folgende Formulierungen wiedergegeben werden kann:

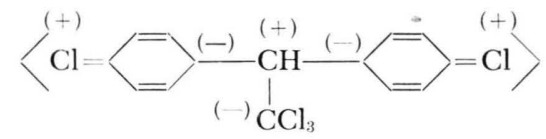

oder

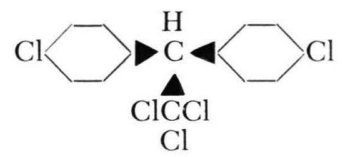

Durch jede Abwandlung im Grundgerüst, die diese Ladungsverteilung verändert, wird gleichzeitig das biologische Wirkungsvermögen verringert oder beseitigt. Man darf daraus schließen, daß zwischen dem Giftmolekül und seinem Wirkungspartner im Organismus ein Komplementär-Verhältnis gleicherweise sterischer wie energetischer Beziehung besteht, wie wir es in ähnlicher Weise zwischen Fermenten und ihren natürlichen Substraten vermuten. Fermente dürften auch beim insektiziden Wirkungsmechanismus primär in Mitleidenschaft gezogen werden. Während eine Fermenthemmung durch das DDT bisher nicht eindeutig nachgewiesen werden konnte, ist sie bei den insektiziden Estern der Phosphorsäure, Thiophosphorsäure und Dithiophosphorsäure experimentell sichergestellt.

Wir wollen im folgenden die insektiziden Verbin- 